\title{
Ferramentas, métodos e experiências no ensino de Engenharia de Software: um mapeamento sistemático
}

Ronnie E. S. Santos ${ }^{1}$, Cleyton V. C. Magalhães ${ }^{1}$, Jorge S. Correia-Neto ${ }^{3}$, Ellen P. R. Souza $^{12}$, Guilherme Vilar ${ }^{3}$

${ }^{1}$ Centro de Informática - Universidade Federal de Pernambuco (CIn/ UFPE) Av. Jornalista Anibal Fernandes- Cidade Universitária, 50670-901 - Recife-PE - Brasil

${ }^{2}$ Unid. Acad. de Serra Talhada - Univ. Federal Rural de Pernambuco (UAST/UFRPE) Fazenda Saco, s/n, Serra Talhada - PE - Brasil

3 Programa de Pós-Graduação em Informática Aplicada (PPGIA) - Universidade Federal Rural de Pernambuco (UFRPE)

Rua Dom Manoel de Medeiros, s/n, Dois Irmãos, 52171-900 - Recife-PE - Brasil

\{ress, cvcm, eprs\}@cin.ufpe.br, jorgecorreianeto@gmail.com, guilherme_vilar@yahoo.com.br

Abstract. Considering that quality of teaching and understanding of themes and contents about Software Engineering have a direct impact in improving software development process, this study aims to identify, analyze and discuss about tool, methods and experiences proposed to support teaching of Software Engineering. To reach this goal, a mapping study was performed and identified 26 research published in two journals and three important conferences on Informatics for education. The papers analyzed mainly discuss about Software management and Software testing.

Resumo. Considerando que qualidade do ensino e a compreensão de conteúdos e temas da engenharia de software tem impacto direto na melhoria do processo de desenvolvimento de software, esta pesquisa tem o intuito identificar, analisar e discutir as ferramentas, métodos e experiências propostas para apoiar o ensino de engenharia de software. Para tal, desenvolveu-se um mapeamento sistemático da literatura, obtendo-se como resultado 26 pesquisas, publicadas em duas revistas e três congressos importantes da área informática na educação, discutindo propostas bastante diversificadas sobre o ensino de engenharia de software, sendo a gerência e o teste de software os tópicos mais abordados.

\section{Introdução}

A engenharia de software, de forma ampla, é a área do conhecimento que fornece toda a estrutura para o desenvolvimento e manutenção de software (IEEE, 2004). Geralmente, a disciplina de engenharia de software é tratada no ensino formal no nível de graduação e/ou pós-graduação ou por meio de treinamentos profissionais de curta duração e, neste contexto, diversas teorias discutem a forma como a engenharia de software deve ser abordada por professores.

Por ser uma importante disciplina da área de Ciência da Computação, muitos defendem a aplicação prática das teorias estudadas de modo a enfatizar a aprendizagem de aspectos tecnológicos, tais como linguagens de especificação, métodos e ferramentas e também os aspectos sociais do desenvolvimento, como a cooperação e a comunicação entre os desenvolvedores (SILVA; LEITE; BREITMAN, 2004). Esta abordagem é coerente com Schneider e Johnston (2003), que discutem a necessidade de trabalhos práticos nas disciplinas de computação e informática para que os alunos consigam assimilar o conhecimento transferido. Segundo Gibbs (1994), a qualidade do ensino e a compreensão dos conteúdos e temas da engenharia de software têm grande impacto na melhoria do processo de desenvolvimento de software e, consequentemente, podem 
apoiar na busca por soluções de problemas e deficiências observadas na prática, no contexto da indústria de software.

Neste sentido, visando consolidar informações quantitativas e qualitativas acerca do ensino de engenharia de software, esta pesquisa tem o intuito identificar, analisar e discutir as ferramentas, métodos e experiências de ensino desta disciplina, realizando um mapeamento sistemático (KITCHENHAM, B.; DYBÅ, T.; JØRGENSEN, 2004) em cinco importantes canais de divulgação da pesquisa sobre informática e educação no Brasil: a) Revista Brasileira de Informática na Educação (RBIE); b) o Simpósio Brasileiro de Informática na Educação (SBIE); c) o Workshop de Informática na Escola (WIE); d) o Workshop sobre Educação em Computação (WEI) e; e) a Revista Novas Tecnologias na Educação (RENOTE).

\section{Procedimentos Metodológicos}

Para realização desse estudo, foi definindo um processo composto por quatro etapas principais, baseado no processo para mapeamento sistemático em engenharia de software aplicado por Petersen et al. (2008) e descritas a seguir.

Etapa 1 - Definição do Escopo do Mapeamento: Compreendeu a construção do protocolo da pesquisa com o planejamento do estudo, definição do processo, das questões de pesquisa, critérios de busca, bem como a composição da equipe. As questões de pesquisa foram divididas em duas facetas, considerando aspectos históricos e conceituais, tais como a evolução histórica das pesquisas brasileiras voltadas para o ensino de engenharia de software e as principais contribuições propostas para o ensino de engenharia de software, respectivamente.

Etapa 2 - Busca e Seleção de Estudos Primários: A busca dos artigos ocorreu de forma automática através de ferramenta de busca disponibilizada no acervo das revistas e de forma manual nas páginas dos congressos. A seleção ocorreu pela análise do título e abstract das publicações. A figura 1 ilustra este processo. A lista completa de estudos incluídos no mapeamento pode ser analisada no Apêndice I desta pesquisa ${ }^{1}$. As pesquisas selecionadas foram identificadas pelo código [ENGXXX].

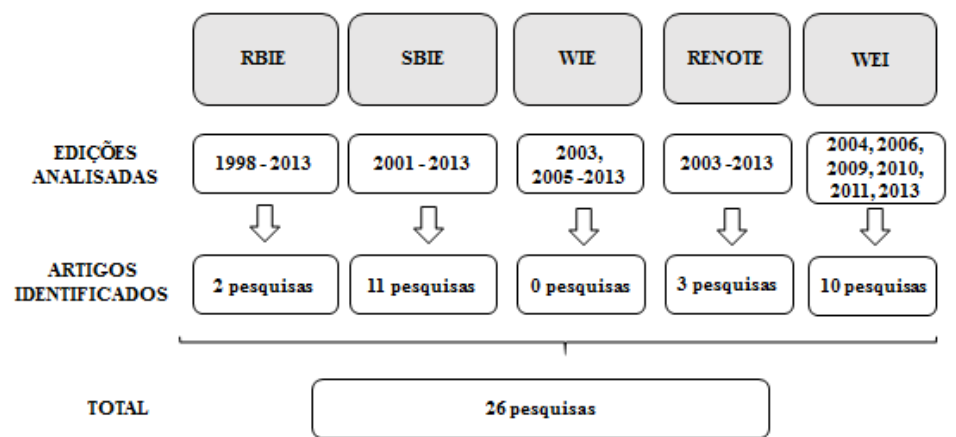

Figura 1. Processo de busca e seleção de estudos.

Etapa 3 - Extração de dados: Nesta etapa, buscou-se extrair informações de cada um dos artigos encontrados, de forma a responder as perguntas de pesquisa. Para auxiliar este processo, um formulário de extração (Tabela 1) foi elaborado utilizando planilhas eletrônicas para extrair das pesquisas informações sobre a identificação do trabalho e

\footnotetext{
${ }^{1}$ Disponível em http://goo.gl/xljRSA.
} 
dos autores, o problema de pesquisa, a proposta, o público alvo para o qual a proposta esteve voltada e a área de concentração da Engenharia de Software, segundo o $\mathrm{SWBOK}^{2}$.

Etapa 4 - Análise e Síntese: A partir dos dados extraídos na fase anterior, deu-se o processo de interpretação dos resultados, criação de tabelas e gráficos para exibição dos dados e descrição das evidências identificadas nos estudos primários selecionados.

\section{Resultados e Discussões}

Esta seção apresenta uma análise descritiva sobre as propostas, que apoiam o ensino de engenharia de software, publicadas nos últimos anos, como apresentado anteriormente na figura 1 .

Aspecto Histórico: Como se deu a evolução histórica das pesquisas brasileiras voltadas para o ensino de engenharia de software?

Em um universo de mais de 1200 publicações, foram identificadas 26 pesquisas com propostas voltadas para o ensino de engenharia de software (figura 2). Deste total $42 \%(11 / 26)$ das pesquisas foram publicadas no SBIE, 38\% (10/26) no WEI, $12 \%(3 / 26)$ na RENOTE e $8 \%(2 / 26)$ na RBIE. Não foi identificada nenhuma pesquisa com essa temática nos anais do WIE. Este acontecimento pode ser explicado pelo próprio foco do evento, que visa principalmente a divulgação de iniciativas nacionais de aplicação das tecnologias da informação e da comunicação nas escolas de ensino fundamental e médio e, de fato, a engenharia de software é uma disciplina presente principalmente em cursos de graduação e de pós-graduação.

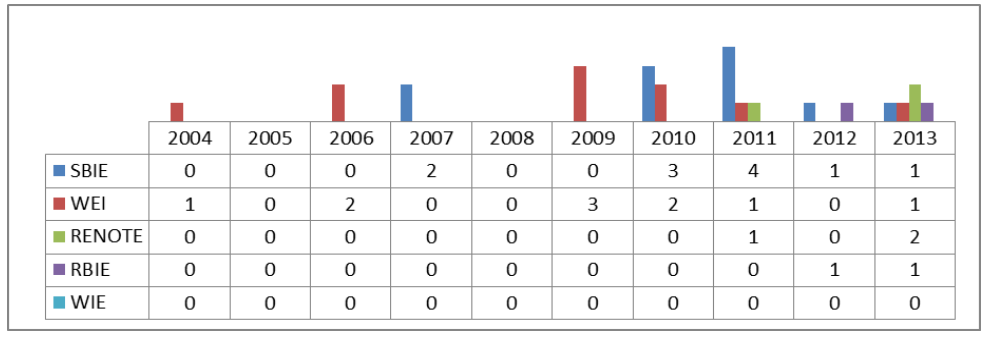

Figura 2. Distribuição anual das pesquisas identificadas.

As pesquisas foram realizadas por 22 instituições diferentes, sendo que em $95 \%$ dos casos (21/22), a pesquisa foi conduzida por professores e pesquisadores de universidades e, em apenas um caso, a pesquisa contou com a participação de um membro de um órgão público. Participaram deste processo universidades de todas as regiões do país, sendo a maior concentração de publicações no sul do Brasil.

Aspectos Conceituais: Quais as principais contribuições propostas para o ensino de engenharia de software?

Os aspectos conceituais das pesquisas identificadas foram analisados segundo três perspectivas: o tipo de proposta da pesquisa (ferramenta computacional, método ou experiência); o tópico da área de engenharia de software abrangido pela proposta (segundo as definições do SWEBOK); e o público alvo para o qual a proposta estava

2 Guide to the Software Engineering Body of Knowledge. Disponível em: http://www.computer.org/portal/web/swebok Acesso em: 12/07/2014. 
mais fortemente voltada. Neste ponto da pesquisa, pode-se considerar para fins de análise 25 dos 26 estudos selecionados. Isto porque não foi possível identificar a versão completa de um dos trabalhos selecionados (ENG023).

Em relação ao tipo de proposta, $48 \%$ das pesquisas $(12 / 25)$ estiveram voltadas para o desenvolvimento de ferramentas computacionais de suporte ao ensino de engenharia de software. Se consideradas as definições de Valente (1999) para software educativo, pode-se observar a presença de três tipos de sistemas educativos: jogo educacional, simulador e software de programação, em alguns casos desenvolvidos de forma combinada, como mostra a tabela 1.

Tabela 1. Classificação das propostas de software educativo, segundo Valente (1999).

\begin{tabular}{cl}
\hline Categoria & \multicolumn{1}{c}{ Pesquisas } \\
\hline Jogo Educacional & {$[$ ENG002][ENG003][ENG005][ENG007][ENG011][ENG014] } \\
& {$[$ ENG016] [ENG026] } \\
\hline Simulador & {$[$ ENG002][ENG003][ENG007][ENG015][ENG025] [ENG026] } \\
\hline Programação & {$[$ ENG010] [ENG019] }
\end{tabular}

Os métodos de ensino estiveram ligados a propostas didáticas e métodos de trabalho sem necessariamente uso de software educativo. Este tipo de proposta foi observado em 32\% dos estudos analisados (8/25). Estas abordagens defendem a necessidade do trabalho em equipe, onde haja um contato real entre os alunos, propício à cooperação e discussões a respeito do desenvolvimento de atividades. Os demais estudos analisados $(20 \%$ - 5/25) não tiveram como objetivo principal uma proposta inédita de nova ferramenta ou novo método para o ensino de engenharia de software. Estas pesquisas apresentaram o relato de experiências de professores ao lecionar esta disciplina em cursos de graduação ou especialização.

Em relação aos tópicos da engenharia de software englobados pelas pesquisas, foi observado que $28 \%$ (7/25) dos estudos identificados pelo mapeamento não tiveram foco específico em um único conteúdo ou tema, englobando os aspectos gerais e questões diversas sobre a área. Enquanto isso, $72 \%$ das pesquisas $(18 / 25)$ estiveram diretamente relacionadas com um tópico específico da área dentre os apresentados no SWEBOK. Entretanto, estas propostas discutiram questões sobre o ensino de apenas cinco dos quinze capítulos sobre engenharia de software englobados pelo guia. Foi possível observar uma grande concentração de pesquisas interessadas principalmente no ensino de gerência de projetos de software e teste de software. A tabela 2 apresenta a distribuição das pesquisas por capítulo do SWEBOK.

Tabela 2. Classificação das propostas de software educativo, segundo Valente (1999).

\begin{tabular}{|c|c|}
\hline Capítulo do SWEBOK & Pesquisas \\
\hline Geral & $\begin{array}{l}\text { [ENG006][ENG008][ENG011][ENG012][ENG017] } \\
\text { [ENG018] [ENG023] }\end{array}$ \\
\hline Software Requirements & {$[$ ENG001][ENG005][ENG009] } \\
\hline Software Construction & [MQUI004] [MQUI020] \\
\hline Software Testing & [ENG007][ENG010][ENG014] [ENG019] [ENG026] \\
\hline Software Engineering Management & $\begin{array}{l}{[\text { ENG002][ENG003][ENG013][ENG015][ENG016] }} \\
{[\text { ENG024] [ENG025] }}\end{array}$ \\
\hline Software Quality & [ENG021] \\
\hline
\end{tabular}

Por fim, foi observado que apenas uma pesquisa teve como público alvo alunos de cursos de especialização; as demais estiveram todas voltadas para os cursos de graduação. Porém, 40\% das pesquisas não apresentaram nenhum tipo de avaliação prática da proposta. Das pesquisas que avaliaram os resultados, em $24 \%$ dos casos essa 
avaliação foi realizada com a participação de alunos de graduação em Ciência da Computação, em 12\% com alunos de graduação em Sistemas de Informação, em 4\% com alunos de graduação em Análise e Desenvolvimento de Sistemas e 4\% com alunos de graduação em Engenharia de Software (4\%). Além disso, 16\% das pesquisas utilizaram alunos de cursos variados da área de Informática. Esta avaliação foi realizada na maioria das vezes através de um método empírico de pesquisa, sendo o estudo de caso o mais utilizado, mas foram encontradas também pesquisas utilizando experimentos controlados e uma pesquisa de campo (survey).

\section{Conclusões e Trabalhos Futuros}

Pode-se dizer que de um ponto de vista geral, ao longo do período analisado foram produzidas pesquisas diversificadas neste contexto, não só com a apresentação de ferramentas computacionais, mas também com métodos e resultados de experiências sobre o ensino da disciplina. Porém ao serem observados aspectos específicos, pode-se concluir que muitos pontos ainda estão em aberto, e os resultados deste mapeamento podem direcionar e encorajar a proposta de novos estudos.

Dentre as questões em aberto pode-se citar o desenvolvimento de outros tipos de software educativo para o ensino de engenharia de software, como os de exercício e prática, os multimídia e os tutoriais (Valente,1999). Além disso, observou-se que há necessidade, pelo menos no contexto brasileiro, de propostas para o ensino dos demais tópicos comumente ensinados nas disciplinas de engenharia de software, tais como processo de software, gerência de configuração, design de software, dentre outros descritos no SWEBOK. Como trabalhos futuros pretende-se estender este mapeamento contemplando pesquisas publicadas em fóruns e encontros realizados dentro de congressos maiores, como o Simpósio Brasileiro de Engenharia de Software (SBES) e o Fórum de Educação em Engenharia de Software e também os congresso e revistas internacionais.

\section{Referências}

IEEE COMPUTER SOCIETY. SWEBOK: guide to the software engineering body of knowledge, 2004.

SCHNEIDER, J., JOHNSTON L. Extreme Programming at Universities - An Educational Perspective. Proceedings of the 25th International Conference on Software Engineering, Portland, Oregon, May 03-10, 2003, p. 594-599.

SILVA, L. F.; LEITE, J. C. S. P.; BREITMAN, K. K. Ensino de Engenharia de Software: Relato de Experiências. Anais do XII Workshop de Educação em Informática (WEI). Salvador, 2004.

GIBBS, W. Software's chronic crisis. Scientific American. 2713 (1994), pp. 86-95.

KITCHENHAM, B.; DYBÅ, T.; JØRGENSEN, M. Evidence-based Software Engineering. 26th International Conference on Software Engineering, (ICSE '04), Proceedings. IEEE, Washington DC, USA, p. 273 - 281, 2004.

PETERSEN, K.; FELDT, R.; MUJTABA, S.; MATTSSON, M. Systematic Mapping Studies in Software Engineering. 12th International Conference on Evaluation and Assessment in Software Engineering (EASE). University of Bari, Italy, 26 - 27 June 2008.

VALENTE, J. A. (1999). Informática na educação no Brasil: Análise e contextualização histórica. In: VALENTE, J.A. (Org.). O Computador na Sociedade do Conhecimento Campinas: UNICAMP/NIED. p. 1-4. 